\title{
SCIENTIFIC REPORTS

\section{OPEN Pioglitazone treatment prior to transplantation improves the efficacy of human mesenchymal stem cells after traumatic brain injury in rats}

Mahasweta Das ${ }^{1,2}$, Karthick Mayilsamy ${ }^{1,2}$, Xiaolan Tang ${ }^{1,2}$, Jung Yeon $\operatorname{Han}^{1,2}$, Elspeth Foran $\mathbb{1}^{1,2}$, Alison E. Willing ${ }^{4}$, Shyam S. Mohapatra ${ }^{1,3}$ \& Subhra Mohapatra ${ }^{1,2}$

Traumatic brain injury is a leading cause of death and disability around the world. So far, drugs are not available to repair brain damage. Human mesenchymal stem cell (hMSC) transplantation therapy is a promising approach, although the inflammatory microenvironment of the injured brain affects the efficacy of transplanted hMSCs. We hypothesize that reducing the inflammation in the cerebral microenvironment by reducing pro-inflammatory chemokines prior to hMSC administration will improve the efficacy of hMSC therapy. In a rat model of lateral fluid percussion injury, combined pioglitazone (PG) and hMSC (combination) treatment showed less anxiety-like behavior and improved sensorimotor responses to a noxious cold stimulus. Significant reduction in brain lesion volume, neurodegeneration, microgliosis and astrogliosis were observed after combination treatment. TBI induced expression of inflammatory chemokine CCL20 and IL1- $\beta$ were significantly decreased in the combination treatment group. Combination treatment significantly increased brain-derived neurotrophic factor (BDNF) level and subventricular zone (SVZ) neurogenesis. Taken together, reducing proinflammatory cytokine expression in the cerebral tissues after TBI by PG administration and prior to hMSC therapy improves the outcome of the therapy in which BDNF could have a role.

Traumatic brain injury (TBI) is a leading cause of death and disability in the United States and around the world. Approximately 10 million people globally either are hospitalized or die from TBI each year ${ }^{1}$. In the United States alone 1.7 million people suffer from TBI and about 52,000 people die $^{2}$. It affects both children and adults and affects the quality of life of the survivors, posing a significant social burden. The most common causes of TBI are related to motor vehicle accidents, sports and battlefield injuries. Falls, especially in children and older people, and physical abuse are among the common causes as well. The National Center for Injury Prevention and Control in the United States recorded 715.7 per 100,000 people TBI-related emergency department visits, 91.7 hospitalizations and 17.1 deaths in $2010^{3}$ with an approximate annual financial burden of $\$ 76.5$ billion $^{3}$. TBI affects the quality of life of the survivors. Even mild TBI or concussions may have effects later in life that are difficult to relate to the prior TBI event. The primary insult to the brain damages the cerebral tissues and the BBB and evokes immune response systemically and locally in the brain. This leads to astrogliosis, immune cell migration, cytokine and chemokine secretion and microglial activation. All these immunological reactions cause further damage to the brain and the secondary injury spreads over time, inducing long term structural, functional, behavioral and psychological deficits.

The available drugs only provide symptomatic treatments to TBI patients. Clinical trials of erythropoietin or progesterone failed to repair brain damage $e^{4-6}$. Transplantation of mesenchymal stromal cells (MSCs) has been

\footnotetext{
${ }^{1}$ James A. Haley Veterans Hospital, University of South Florida College of Medicine, Tampa, FL, 33612, USA. ${ }^{2}$ Department of Molecular Medicine, University of South Florida College of Medicine, Tampa, FL, 33612, USA. ${ }^{3}$ Department of Internal Medicine, University of South Florida College of Medicine, Tampa, FL, 33612, USA. ${ }^{4}$ Department of Neurosurgery and Brain Repair, University of South Florida College of Medicine, Tampa, FL, 33612, USA. Correspondence and requests for materials should be addressed to S.M. (email: smohapa2@health.usf.edu)
} 
shown to be the most promising regenerative approach. These pluripotent cells have the potential to be transformed into many different cells, are immune suppressive and secrete growth factors to promote tissue regeneration $^{7-9}$. In addition to time of transplantation, number and quality of transplanted cells, the efficacy of MSC therapy largely depends on the microenvironment of the target tissue. Following TBI, proinflammatory cytokines are secreted in the brain parenchyma, immune cells migrate and glial cells are activated creating an inflammatory environment which may not favor the survival and functioning of the MSCs ${ }^{10}$. Therefore, reducing the inflammation before MSC therapy might help to increase the efficacy of the therapy.

We hypothesize that reducing proinflammatory cytokine production in brain tissue will enhance the efficacy of hMSC treatment and improve the histopathological and behavioral outcomes in rat after TBI. Previously we have shown that the proinflammatory chemokine CCL20 is produced in the degenerating cerebral tissues after $\mathrm{TBI}^{11,12}$. Pioglitazone is a peroxisome proliferator-activated receptor gamma (PPAR $\gamma$ ) agonist belonging to the thiazolidinedione (TZD) class of drugs and used to treat hyperglycemia. When used in low dose it showed a cardio-protective property in experimental animals ${ }^{13}$. Qi et al. (2011) have shown that PPAR $\gamma$ negatively regulates CCL20 expression in the renal epithelium ${ }^{14}$. PG has also been shown to reduce proinflammatory cytokines IL1- $\beta$ and IL- 6 in different tissues ${ }^{15-17}$. Therefore, in this study we chose to use PPAR $\gamma$ agonist pioglitazone (PG) to reduce TBI-induced cytokine production in the cerebral tissues and thereby, improve the microenvironment for hMSC functioning after TBI.

\section{Results}

Intra-nasally administered hMSCs were delivered to the brain post TBI. Intranasal (i.n.) delivery of hMSCs to the brain was confirmed in a subset of rats. DiR labeled hMSCs were delivered through i.n. or intravenous (i.v.) routes. IVIS imaging showed the presence of DiR fluorescence in brain, lung, liver and spleen 7 days after hMSC administration (Fig. 1A,B). The i.n. delivery showed significantly higher DiR signal in the brain compared to i.v. delivery (Fig. 2B). DiR fluorescence indicates that a large portion of the hMSCs delivered by i.v. route was cleared by the system in 7 days, whereas, the i.n. delivered hMSCs stayed in the system. Presence of hMSCs in the brain 35-days post impact (dpi) was confirmed by immunohistochemical staining of the brain sections with anti-human nuclear antigen (clone 235-1) antibody (HuNu). The immunostaining showed the existence of HuNu positive nuclei in the ipsilateral cortex close to the lesion area in hMSC or combination treated rats (Fig. 1C). In addition, a few scattered HuNu-DAPI double positive cells were observed in the treated brains. The number of HuNu positive cells in the perilesional area of the brains observed at $7 \mathrm{dpi}$ did not change significantly at $35 \mathrm{dpi}$ (Fig. 1D).

Combination treatment reduced the pathological changes and neurodegeneration in the rat brain cortex. Thionin staining clearly shows tissue loss and decreased cellularity in the TBI and vehicle treated animals as compared to sham. Significant cortical tissue loss was evident after 35 dpi. Lesion volume in the vehicle treated group was not significantly different from the TBI alone group. PG treated animals showed some reduction in the lesion volume but this was not statistically significantly different from lesion volume in the TBI alone group. On the other hand, lesion volume decreased significantly after hMSC or combination treatment (Fig. 2A,B).

Neurodegeneration is evident in the TBI animals 35 dpi. FJ staining in the rat brain shows damaged FJ positive cells and fibers in the ipsilateral cortex around the impact site and the lateral part of the cortex, around the primary sensorimotor area in TBI and the vehicle treated groups. White matter damage was also visible in these brains in the ipsilateral corpus callosum and striatum. The treated groups (PG, hMSC or combination) showed significantly fewer degenerating cells or fibers than the TBI or vehicle groups, but there was no significant difference among the treatment groups (Fig. 2A-FJ panel, B). FJ positive fibers were also observed in corpus callosum in the regions adjacent to the degenerated areas in TBI or vehicle treated groups. Some FJ positivity was also observed in the PG or hMSC treated animals but not in the combination treated group (Fig. S1). Contralateral brain did not show cellular or fiber damage in animals from any of the groups.

Effect of combination treatment on neuroinflammation. Microgliosis. Microgliosis, as indicated by an increased number of Ibal positive microglia in the cerebral tissue, was observed $35 \mathrm{dpi}$ in TBI, as well as vehicle treated animals. The number of Ibal positive microglia significantly increased in TBI animals as compared to sham. Increased number of microglia were observed in the ipsilateral cortex (adjacent to the injury site) (Fig. 2 A-Iba1 panel, B-Iba1 + cells), lateral part of the cortex and corpus callosum (Fig. S1A). All the treatment groups showed significantly fewer Ibal positive microglia in these areas compared to TBI or vehicle treated groups in the cortex, and the combination treatment group showed significantly fewer Ibal positive cells compared to TBI, vehicle or PG treated animals (Fig. 2 B-Iba1 + cells).

The number of Ibal positive cells also increased in the contralateral hemisphere of these areas but not as much as the corresponding areas of the ipsilateral hemisphere (Fig. S2). In the contralateral corpus callosum also, combination treated animals showed significantly fewer Ibal positive cells as compared to TBI, vehicle, PG or hMSC groups.

Astrogliosis. Increased GFAP immunoreactivity with scar formation was observed in all other groups as compared to sham (Fig. 2A- GFAP panel, B-GFAP integrated density) indicating activation of astrocytes 35 dpi. In the TBI and vehicle groups, astroglial activation and glial scars were still observed after $35 \mathrm{dpi}$. PG treatment reduced the GFAP immunoreactivity significantly as compared to TBI or vehicle treated animals. In hMSC treated animals, the GFAP immunoreactivity increased significantly compared to TBI, vehicle or PG treated groups, although close observation did not reveal glial scar formation in these animals. In the combination treated animals, GFAP immunoreactivity was high, although not significantly, compared to the TBI or vehicle groups, and no glial scars were observed in these brain sections. On the other hand, in this group, GFAP immunoreactivity was significantly reduced from the hMSC treated group (Fig. 2B-GFAP integrated density). 


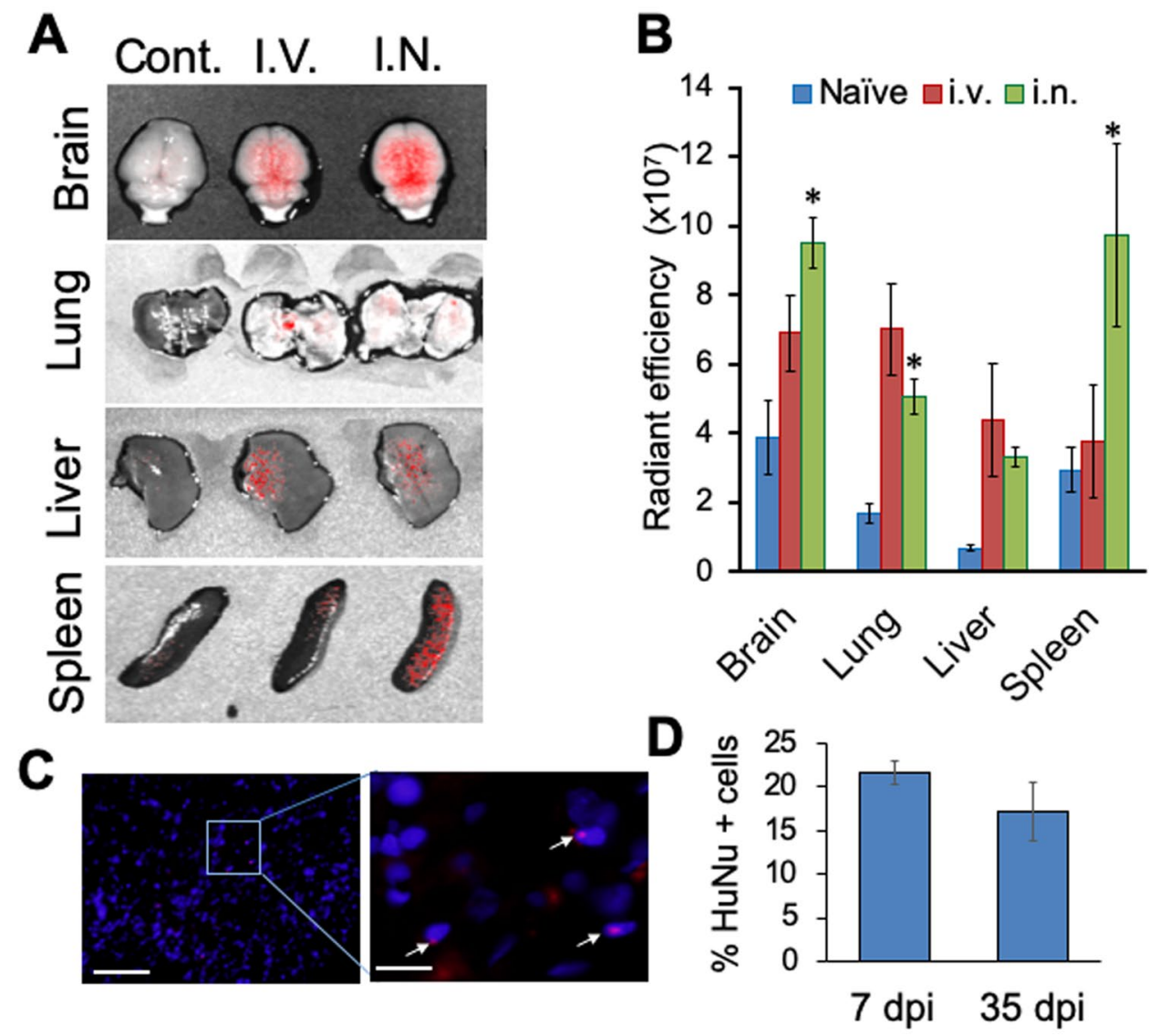

Figure 1. Delivery of hMSC after TBI. (A) Biodistribution of hMSCs after TBI. Representative Xenogen images showing DiR fluorescence in different organs 7 days after TBI. (B) Histogram showing the significantly higher level of $\mathrm{DiR}$ fluorescence in brain and spleen following intra-nasal (i.n.) administration as compared to intravenous (i.v.) administration, $\mathrm{n}=3$ animals/group. $* \mathrm{p}<0.01$ vs i.v. (C) Brain sections from rats after 35 days of TBI were stained with anti-human nuclear antigen ( $\mathrm{HuNu}$ ) antibody (clone 235-1). The immunostaining indicates the presence of hMSCs (arrows) in the ipsilateral cortex close to the lesion site. Scale bar $100 \mu$, inset scale bar $20 \mu$. (D) Histogram showing the percentage of $\mathrm{HuNu}$ positive cells in the perilesional area of the brains. HuNu positive cells which were also DAPI positive were counted from the perilesional area of each section located $120 \mu$ apart. Also, 50 DAPI positive cells were counted from the same area of the sections. $\%$ of $\mathrm{HuNu}$ positive cells was calculated from $7 \mathrm{dpi}$ and $35 \mathrm{dpi}$ brains as shown in the histogram. A paired test did not show any significant difference between the $\%$ of $\mathrm{HuNu}$ positive cells present in the brains of $7 \mathrm{dpi}$ or $35 \mathrm{dpi}$. $\mathrm{n}=3$ (7 dpi), and 7 (35 dpi).

Cytokine expression. Expression of CCL20 is a landmark of neuroinflammation after TBI. After 35 dpi, TBI and vehicle treated animals express significantly more CCL20 in the cortex compared to sham (Fig. 3A,B). In our pilot study, we observed that CCL20 expression was up-regulated and PPAR $\gamma$ was downregulated in the brain $48 \mathrm{~h}$ post-TBI (Fig. S3). Also, PG treatment reduced CCL20 expression and increased PPAR $\gamma$ expression $48 \mathrm{~h}$ post TBI (Fig. S3). In the present study, PG treatment reduced CCL20 expression 35 dpi. Both hMSC treatment, as well as combination treatment, reduced CCL20 expression in the cortex. Importantly, CCL20 expression in the ipsilateral cortex in the combination treatment group was significantly lower than that in hMSC treatment group indicating better efficacy of the combination treatment (Fig. 3A,B). The expression of IL1- $\beta$ in the ipsilateral cortex was also increased in the TBI or vehicle treated groups and decreased after PG, hMSC or combination treatment. IL1- $\beta$ expression decreased significantly in the combination treated group compared to hMSC treated group (Fig. 3A,C). This cytokine expression profile clearly indicates that PG treatment prior to hMSC therapy reduced the proinflammatory microenvironment facilitating the performance of hMSC.

PG-hMSC combination treatment increased neurogenesis. Neurogenesis is an important indicator of recovery from TBI. Neurogenesis occurs at a basal level in the adult, uninjured animals (sham) in the sub-ventricular zone (SVZ) area of the brain as observed in our experiment. In TBI or vehicle treated animals, neurogenesis significantly decreased as indicated by decreased DCX immunoreactivity. PG or hMSC treatments increased neurogenesis but not significantly compared to TBI or vehicle treated animals, whereas combination treatment significantly increased neurogenesis in the SVZ region compared to TBI or vehicle groups. In the combination group, neurogenesis was restored to the same extent observed in sham animals (Fig. 4A,B). 


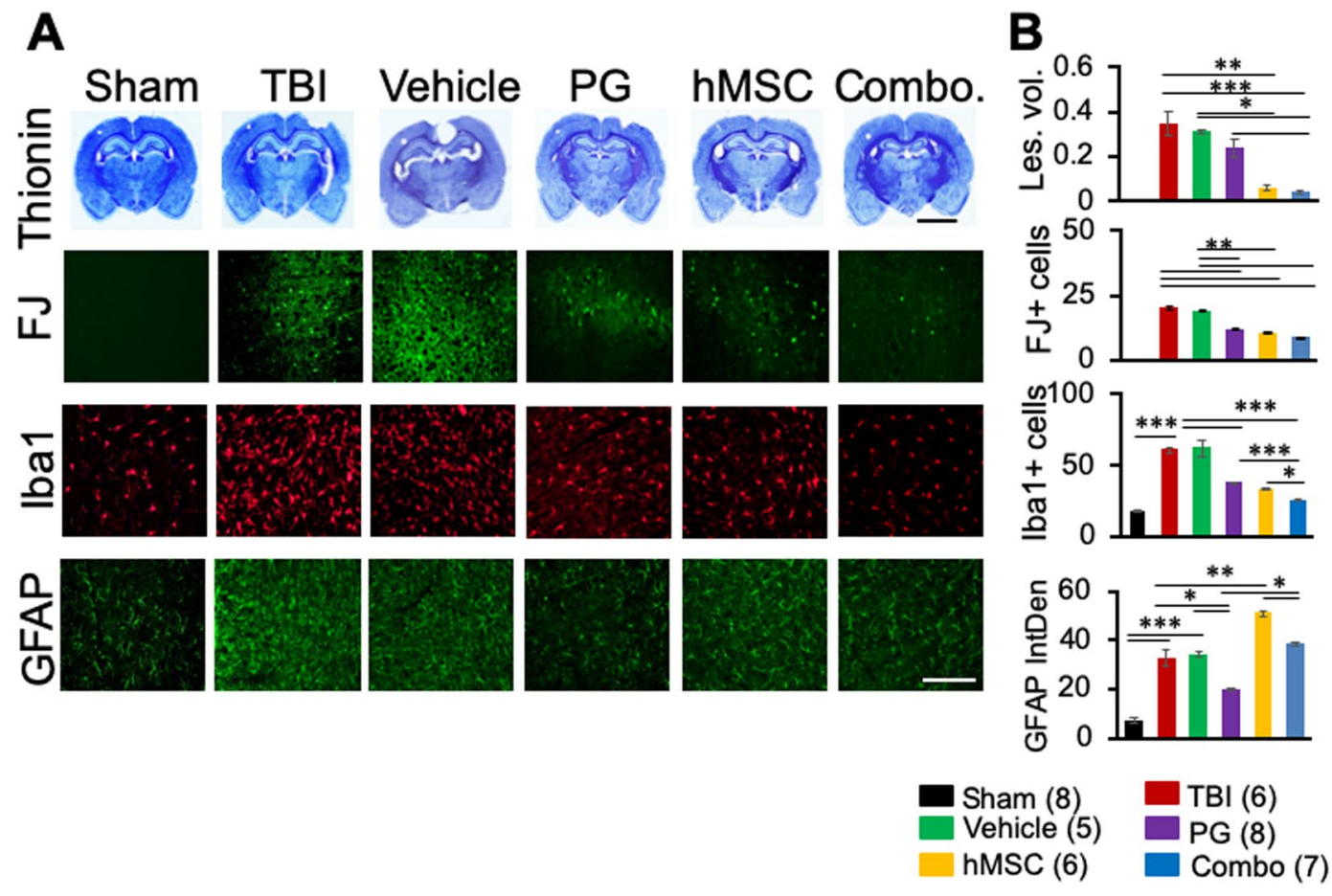

Figure 2. hMSC or PG + hMSC combination treatments reduce brain damage and neuroinflammation 35 days after TBI. Representative images of brain sections (A) or histograms showing image J quantitation. (B) Thionin images are low magnification images (scale bar $500 \mu$ ), showing tissue loss. The average tissue loss is expressed as lesion volume $\left(\mathrm{mm}^{3}\right)$ in the histogram. Remaining panels in A show degenerating cells and fibers (FJ panel), microgliosis (Ibal panel) or astrogliosis (GFAP panel) in the ipsilateral cortex 35 days after TBI. Lesion volume was measured in $\mathrm{mm}^{3}, \mathrm{FJ}$ and Iba1 were measured by counting positive cells and GFAP was measured by integrated density using image J. Les vol., lesion volume, PG, pioglitazone, hMSC, human mesenchymal stem cells, combo., $\mathrm{PG}+$ hMSC combination treatment. Numbers in the parenthesis indicate the number of animals in each group. $* \mathrm{p}<0.01, * * \mathrm{p}<0.001$ vs TBI, $* * * \mathrm{p}<0.0001$. Scale bar $100 \mu$.

PG-hMSC combination treatment increased brain-derived neurotrophic factor (BDNF) secretion. The neurotrophic factor BDNF supports neurogenesis. We observed that serum BDNF level, as measured by ELISA, decreased in TBI and vehicle treated groups. PG treatment restored the level close to sham animals while hMSC and combination treatment significantly increased serum BDNF level. BDNF level after combination treatment was significantly higher than in the hMSC treated group (Fig. 4C).

Combination treatment reduced the anxiety-like behavior of TBI rats in the open field. Behavior in the Center Zone (CZ). Rat behavior in the open field arena before and after TBI was recorded and analyzed using Noldus Ethovision software as described in the methods section. A heat map was generated showing the activities in the arena (Fig. 5A). As a general tendency, rats preferred to stay close to the walls of the apparatus. The baseline (Day 0 ) behavior showed that the rats were visiting the $\mathrm{CZ}$ frequently while staying mostly to the peripheral zone close to the wall of the open field box. The 35 days recording showed changes in the open field behavior of the rats. Both TBI and vehicle treated animals avoided the $\mathrm{CZ}$ of the arena, did not spend time in the $\mathrm{CZ}$ and when they explored the $\mathrm{CZ}$ for the first time, had long latency (Fig. $5 \mathrm{~A}-\mathrm{C}$ ). On the other hand, as seen in the heat maps, PG, hMSC or combination treated rats explored the CZ more frequently (Fig. 5A), had lower latency to the first entry to the $\mathrm{CZ}$ (Fig. $5 \mathrm{~B}$ ), spent more time in the $\mathrm{CZ}$ (Fig. $5 \mathrm{C}$ ) and entered the $\mathrm{CZ}$ more frequently (Fig. 5D).

In the open field arena rats were busy in travelling, rearing and occasional grooming. We did not observe significant difference in their locomotor activities in the open field. The distance traveled or the movement velocity were not significantly different between groups indicating that TBI did not affect the locomotor behavior of the rats (Fig. S4).

Immobility in the open field arena. Analysis of other anxiety-like behaviors in the open field showed that the TBI and vehicle treated animals had a shorter latency to become immobile (Fig. 5E), exhibiting anxiety-like behavior in the open field. This behavior improved significantly after the combination treatment as the combination group showed the longest latency to become immobile indicating that they were least anxious in the open field. Combination treatment significantly improved the anxiety-like behavior over the hMSC or PG treated groups (Fig. 5E). 


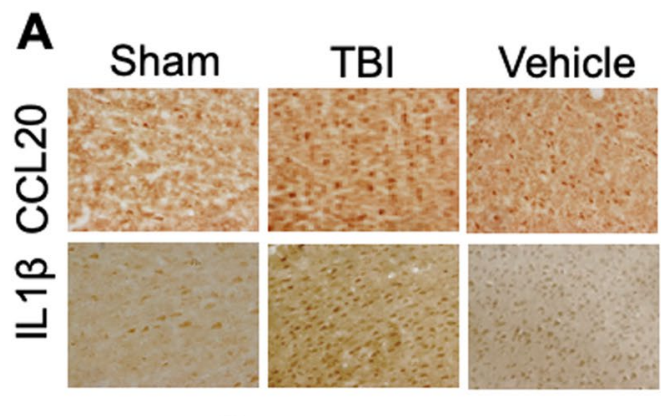

\section{B}

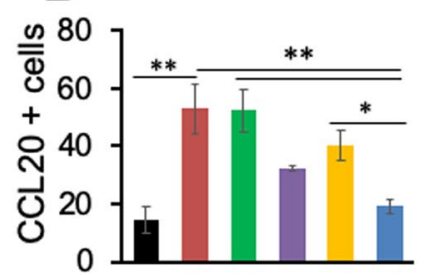

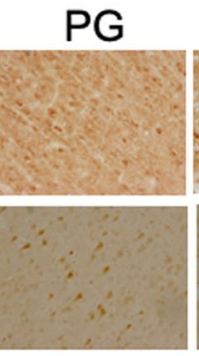

hMSC
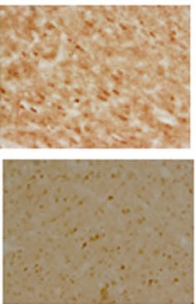

C

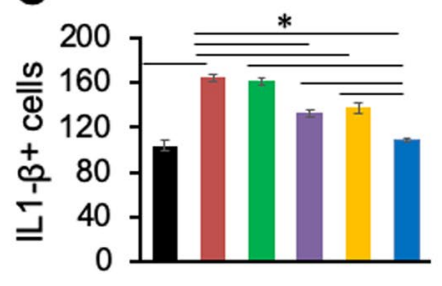

Sham (8)

TBI (6)

Vehicle (5)

PG (8)

hMSC (6)

Combo(7)

Figure 3. Combination treatment significantly decreases the expression of inflammatory chemokines CCL20 and IL1- $\beta$ in the rat brain cortex 35 days after TBI. (A) Representative images of immunoperoxidase staining of cortex (adjacent to the injury site) showing the CCL20 or IL1- $\beta$ immunoreactivity under different experimental conditions. Scale bar $100 \mu$. (B,C) Histograms showing the numbers (mean \pm SEM) of CCL20 (B) or IL1 $\beta$ (C) positive cells in the cortex. PG, pioglitazone, hMSC, human mesenchymal stem cells, combo., PG + hMSC combination treatment. Numbers in parentheses indicate the number of animals in each group. ${ }^{*} \mathrm{p}<0.01$, $* * \mathrm{p}<0.001$.

A

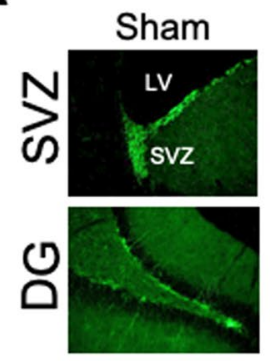

TBI

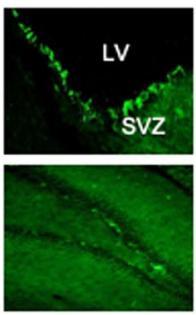

B

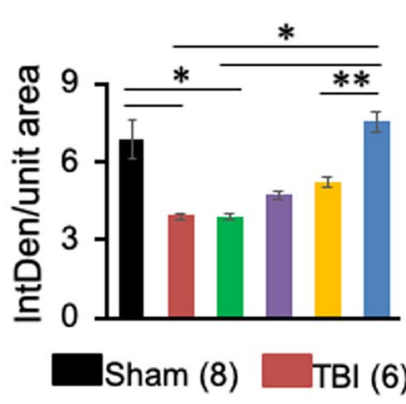

DMSO
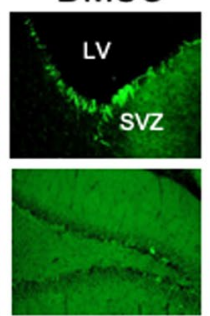

C

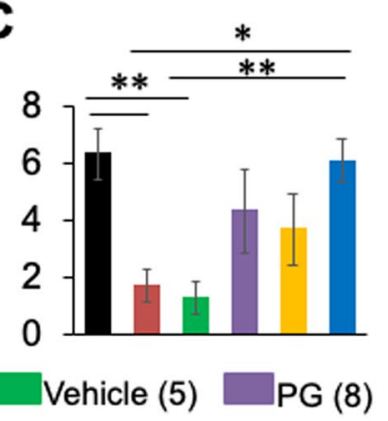

hMSC

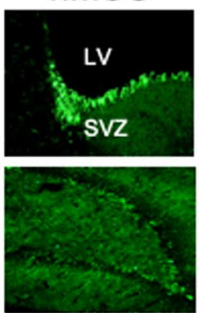

D

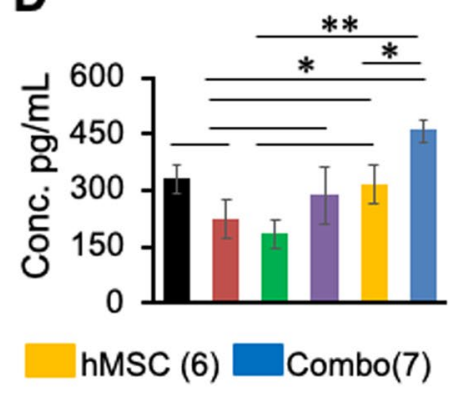

Figure 4. PG-hMSC combination treatment improves neurogenesis and increases BDNF level after TBI. (A) Representative immunofluorescence images showing DCX immunoreactivity in the SVZ (Scale bar $100 \mu$ ) or DG region (Scale bar $200 \mu$ ) under different experimental conditions. (B) Histogram showing DCX immunoreactivity (Mean \pm SEM) in SVZ (B) or DG (C) under different experimental conditions measured using image J. (D) Histogram showing serum BDNF level measured by ELISA. IntDen, integrated density, PG, pioglitazone, hMSC, human mesenchymal stem cells, SVZ, subventricular zone, LV, lateral ventricle, DG, dentate gyrus. Numbers in parentheses indicate the number of animals in each group. ${ }^{*} \mathrm{p}<0.01,{ }^{* *} \mathrm{p}<0.001$. 


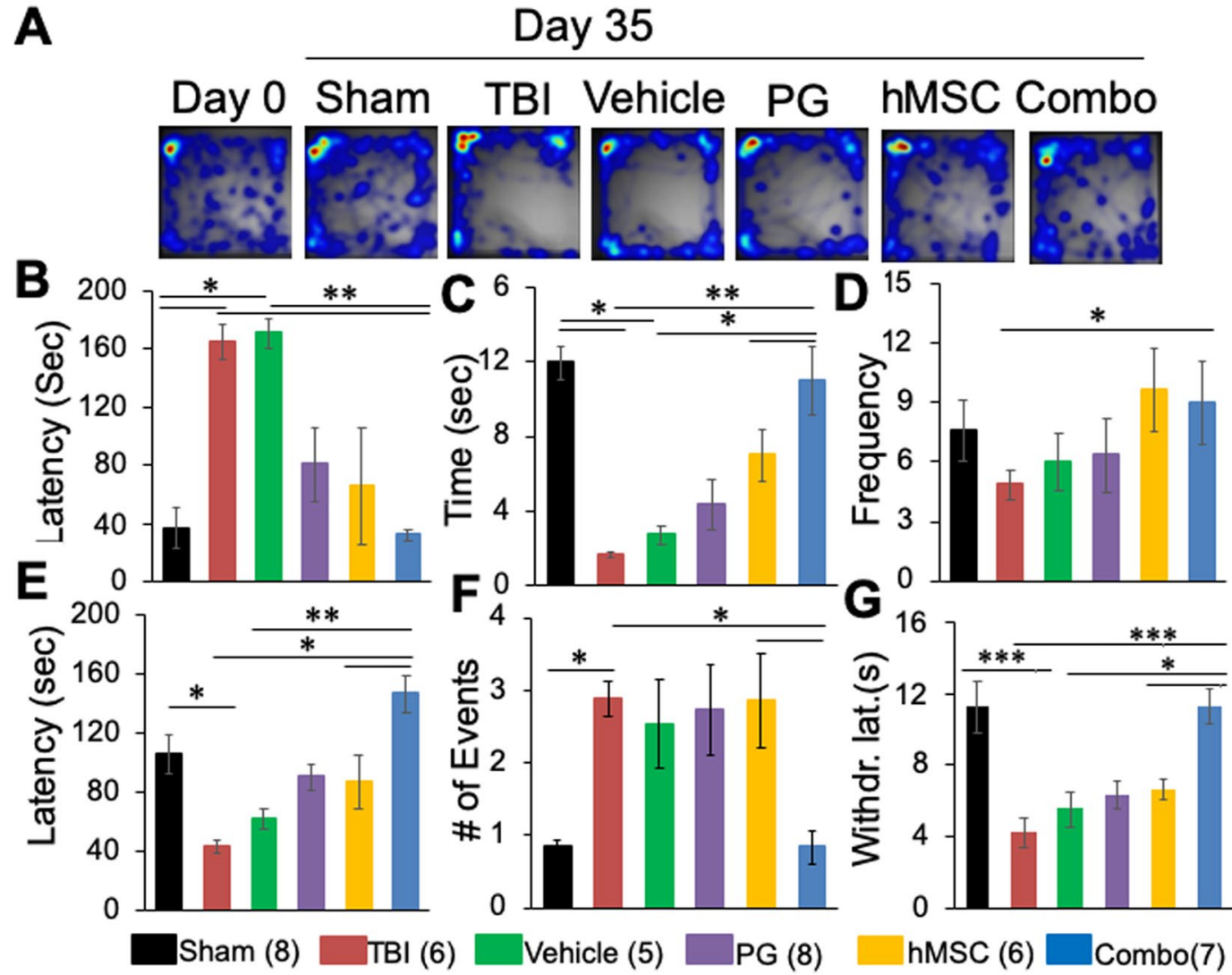

Figure 5. Open field or sensorimotor behavior of rats under different experimental conditions. (A) Representative heat maps of the movement of rats in the open field arena. Baseline behavior (Day 0) was recorded before TBI or sham surgeries. Histograms show the average values (mean \pm SEM) of anxiety-like behaviors of rats in the open field arena. (B-F) Anxiety-like behavior of rats in the open field, (B) Latency to the first entry to the center zone of the arena, (C) Time spent in the center zone of the arena, (D) Frequency to entry to the center zone, (E) Latency to first immobility in the arena, (F) Number of grooming events. (G) Mean paw withdrawal latency $(\mathrm{sec})$ of the contralateral paw to noxious cold stimulation (mean $\pm \mathrm{SEM}$ ). $\mathrm{PG}$, pioglitazone, hMSC, human mesenchymal stem cells, Combo, PG + hMSC combination treatment. Numbers in parentheses indicate the number of animals in each group. ${ }^{*} \mathrm{p}<0.05, * * \mathrm{p}<0.01, * * * \mathrm{p}<0.001$.

Grooming and rearing behavior. TBI and vehicle treated rats showed a significantly increased number of grooming events compared to sham. PG or hMSC treatment did not alter this behavior. However, combination treatment reduced the number of grooming events significantly compared to TBI. The number of grooming events was significantly lower in the hMSC group indicating an improvement in behavior (Fig. 5F).

Combination treatment improved the TBI induced hyperalgesia. The cold plantar assay showed a significant decrease in the withdrawal latency of the contralateral paw in the TBI or vehicle treated rats compared with sham (Fig. 5G). The combination treatment group had a significant increase in withdrawal latency compared to the control groups (TBI and vehicle). Also, we observed that in the combination treatment group, withdrawal latency increased significantly from the hMSC treated group (Fig. 5B). However, we did not see any significant difference in withdrawal latency in the ipsilateral paw. This observation indicates that the combination treatment helped in recovering from TBI-induced hyperalgesia.

\section{Discussion}

In this study, we have observed significant tissue loss, degeneration of neurons and neuronal fibers in several brain areas $35 \mathrm{dpi}$, indicating secondary spread of the damage. Presence of Ibal positive activated microglia, intense astrogliosis and presence of glial scars along with an elevated expression of proinflammatory chemokine CCL20 and IL1- $\beta$ indicate persistent active inflammation in the brain after TBI in TBI and vehicle treated animals $35 \mathrm{dpi}$. This is further supported by decreased BDNF in serum after TBI.

For recovery from TBI, it is important to not only restore the damaged tissues but also to prevent the spread of secondary neurodegeneration. Attempts have been made to treat this condition with drugs, but drugs like erythropoietin ${ }^{4,5}$ and progesterone $e^{5,6}$ have failed at clinical trials despite their neuroprotective properties. Recently MSCs have become the focus of a regenerative approach in the treatment of TBI. Several investigators have shown the potential of MSCs in cerebral regeneration after TBI. These pluripotent cells are present in a wide variety of body tissues ${ }^{18,19}$ and are easy to isolate and culture and potentially, they can differentiate into many 
kinds of cells ${ }^{20-22}$. Their immune tolerance ${ }^{7}$, ability to migrate to the site of inflammation ${ }^{23-25}$, and secretion of growth-promoting factors ${ }^{7-9}$ makes them an attractive candidate for regenerative therapies. In our study, we have observed that hMSC treatment improved the behavioral outcome in rats, reduced the brain lesion volume, neuronal death, microglial activation, and CCL20 and IL1- $\beta$ expression. On the other hand, it increased the expression of BDNF and neurogenesis. Together these observations indicate an overall trend towards the reduction of post-TBI inflammation and increased regeneration. Increased neurogenesis in the hMSC treated animals shows the regenerative potential of these cells but neurogenesis did not increase to the level as observed in the sham animals. Therefore, the expected outcome was not achieved by hMSC treatment alone. On the other hand, the hMSC induced modulations in behavioral, immunological and regenerative outcomes were significantly enhanced by PG treatment prior to hMSC transplantation, i.e., by the combination treatment.

The efficacy of hMSC treatment depends upon a few factors such as the number of administered cells, route of administration, time of administration and the microenvironment of the target tissue. Keeping these factors in mind, we administered an optimum number of cells $\left(1 \times 10^{6} \text { cells per animal }\right)^{26}$ through the intranasal route. Studies have shown that i.v., intra-arterial (i.a.) or intra-cerebral routes have potential disadvantages. Xiong et al. showed that i.v. administration of MSCs caused systemic distribution while i.a. administration caused cerebral ischemia and intracerebral administration limits the number of injected cells to a sub-optimal dose ${ }^{27}$. On the other hand, the i.n. route used in this study is the easiest non-invasive delivery route. Bypassing the BBB, the cells enter the cerebral parenchyma through the nasal mucosa and the cribriform plate ${ }^{28,29}$ and reach the brain within 1 hour of administration ${ }^{30}$. In our pilot experiments, we administered DiR-labelled hMSCs via i.v. or i.n. route. We observed significantly higher DiR fluorescence in the brain after i.n. administration compared to the i.v. group (Fig. 1A,B), indicating higher efficacy of this method. In addition, we also observed the presence of the administered cells in the treated brains $35 \mathrm{dpi}$. The percentage of hMSCs surviving at 35 dpi was not significantly less than the percentage of cells observed at $7 \mathrm{dpi}$ indicating successful transplantation, homing, and survival of cells after i.n. administration. We, therefore, followed this method of hMSC administration throughout this study.

The success of hMSC transplantation therapy also depends on the microenvironment of the recipient tissue. Garcia-Olmo et al. have shown that the low success rate of the stem cell transplantation therapy owed to the proinflammatory cytokines and reactive oxygen species secreted by the immune cells in the inflammatory microenvironment of the injured tissue ${ }^{10,31}$. The caspases secreted in this environment caused apoptosis of the transplanted cells, leading to a low success rate of the therapy ${ }^{32}$. Impaired survival and homing efficacy of stem cells due to post-trauma inflammation have been reported by Malcanyi et al. ${ }^{33}$. In an attempt to improve the regenerative efficacy of the stem cells in a mouse skin wound model, Geesala et al. (2017) transplanted stem cells with or without the oral cyclooxygenase (COX)-2 inhibitor, celecoxib. In this study, they observed increased engraftment and differentiation of stem cells leading to enhanced wound tissue repair when the cells were transplanted with celecoxib $^{34}$. PG, belongs to the group of thiazolidinediones (TZDs) which are synthetic high-affinity ligands of PPAR $\gamma$; it is a potent anti-inflammatory drug known to reduce the production of inflammatory cytokines after brain injury ${ }^{35-37}$, including IL1- $\beta$ and IL6 ${ }^{15-17}$, tumor necrosis factor $\alpha$ (TNF $\alpha$ ), inducible nitric oxide synthase (iNOS), matrix metalloproteinase (MMP)-9 and COX-2 in macrophage, glial cells, and neurons ${ }^{38}$. It has been shown to inhibit CCL20 production, thereby reducing macrophage infiltration and resulting inflammation ${ }^{14}$. It is hypoglycemic ${ }^{14}$, immunomodulatory ${ }^{16}$ and in low doses, it is cardio-protective ${ }^{13}$. It acts on numerous target genes and pathways including the canonical WNT/ $\beta$-catenin pathway and represses nuclear factor $\kappa \mathrm{B}(\mathrm{NF}-\kappa \mathrm{B})$ and $\mathrm{TNF} \alpha$, thereby interfering with the pro-inflammatory pathway ${ }^{39}$. In our preliminary study, we observed that PPAR $\gamma$ and CCL20 expression were inversely correlated in the brain. High expression of PPAR $\gamma$ was observed in the brain in the absence of CCL20 expression in sham animals. On the other hand, in TBI animals, PPAR $\gamma$ expression declined with the increased expression of CCL20 (Fig. S3). We also observed PG treatment reduced CCL20 expression post-TBI (Fig. 3). These preliminary observations along with information from published literature prompted us to use PG as the anti-inflammatory drug of choice for this study. We treated the animals with PG following TBI and continued the treatment until hMSC transplantation. This therapeutic strategy enhanced the efficacy of hMSC therapy in decreasing post-TBI inflammation, improving neurotrophic factor secretion and neurogenesis. All these effects were reflected in improved behavioral and sensorimotor responses. The overall treatment outcome of the combination treatment was significantly improved over PG only or hMSC only treatments. Although both hMSC and the combination treatment reduced the lesion and neurodegeneration to a similar extent, the combination treatment was more efficient in reducing the microglial activation in all brain regions examined (Figs 2, S1), even on the contralateral side (Fig. S2). Inflammatory mediators like CCL20 and IL1- $\beta$ were also significantly reduced by the combination treatment, indicating the efficacy of this strategy over the other treatment options.

Depression and anxiety disorders and locomotor deficits are common and devastating aftereffects of TBI. In this study, we did not observe motor deficits in the rats $35 \mathrm{dpi}$ as there was no significant change in their locomotor activity in the open field (Fig. S4). Although generally considered psychological, anxiety and depression may have neurobiological underpinnings. The open field test in rodents is a simple, yet promising, test to evaluate such neurobehavioral aspects of TBI. In an open field arena, rats typically prefer to be close to the walls, a behavior called thigmotaxis. They tend to avoid the open, well-lit area of the center which is a novel, stressful environment to the animal while making occasional exploratory trips to the center area. When the animals are less anxious they tend to spend more time in the open center zone and also decrease the latency to enter this area (anxiolytic activity). Jones et al. (2008) showed that 1 to 3 months after TBI induced by fluid percussion injury (FPI), rats showed anxiety-like behavior by exhibiting reduced entry and reduced time spent in the center area of an open field arena ${ }^{40}$. Recently, Kim et al. have shown increased activity in the center zone of rats treated with the synthetic estrogen, $17 \alpha$-ethynylestradiol-3-sulfate, after FPI-induced $\mathrm{TBI}^{41}$. In line with these reports, our findings indicate that TBI and vehicle treated rats showed significant anxiety-like behavior and this behavior improved 
in the combination treatment group over other treatment groups. The rats with combination treatment showed lower latency to enter the $\mathrm{CZ}$, they spent more time in the $\mathrm{CZ}$ and entered the zone more frequently indicating anxiolytic behavior. They also exhibited the longest latency to first immobility and the least grooming. All these behaviors indicated that the functional recovery was best in the combination treated rats.

Sensory hypersensitivity and persistent pain, including headache, nociceptive pain, and neuropathic pain are somatic symptoms of $\mathrm{TBI}^{42}$. Pain has been reported as a secondary complication of $\mathrm{TBI}^{43,44}$. Very few models are available that describe post-injury pain and its mechanism ${ }^{45,46}$. In the current study, we have shown increased thermal pain sensitivity (decreased withdrawal latency to noxious cold exposure) in the contralateral paw in rats after TBI. This indicates the involvement of the central sensorimotor pathway in this phenomenon. Following TBI, synthesis and release of inflammatory cytokines and pro-nociceptive mediators from activated microglia have been reported ${ }^{47}$. Rowe et al. implicated glial activation, central and peripheral inflammatory mediators and $\mathrm{T}_{\text {reg }}$ dysregulation as potential causes of TBI-induced mechanical hyperalgesia ${ }^{42}$. In our study, combination treatment has shown to reduce the hyperalgesia to the normal level. It is possible that this mechanism is correlated to the reduction of cerebral neuroinflammation, tissue regeneration and re-establishment of the neural connections by the combination treatment after TBI. Further investigation is needed to elucidate the actual mechanism of combination treatment induced alleviation of sensorimotor response to noxious cold sensation after TBI. Regardless of the mechanism, our observations strongly suggest a behavioral and sensorimotor recovery in the combination treatment group after TBI.

We observed that hMSC or combination treatment increased GFAP immunoreactivity, although no glial scar formation was observed in the treated groups. The astrocytes in these groups maintained their territory all over the brain. At the same time, CCL20 decreased in these groups indicating the reduced inflammatory status of the brain. Astrocytes are an important cellular component in maintaining the structure and functions of CNS. They are essential for neuronal survival ${ }^{48}$. Reactive astrogliosis is indicative of injury or insult to the brain $^{49}$. They can be neuroprotective or neurotoxic ${ }^{49,50}$. Following TBI, astrocytes help in restricting the spread of damage by forming glial scars ${ }^{20}$. It has been shown that MSCs release chemokines which activate surrounding astrocytes facilitating tissue repair ${ }^{20,51}$. Following administration after TBI, MSCs have migrated to a distant location from the lesion site and differentiated into neurons and astrocytes improving motor functions ${ }^{20}$. It is possible that in our study, astrocytosis in the hMSC or combination treated animals is actually neuroprotective or regenerative. More research is needed to elucidate the exact role played by the astrocytes under the present circumstances.

Neurotrophic factors are associated with reactive processes occurring as a result of lesions in the CNS. BDNF is a neurotrophic factor which has been shown to be associated with post-TBI depression and cognitive dysfunction ${ }^{52,53}$. It is also involved in neuronal survival and synaptic plasticity ${ }^{54,55}$. Following TBI, a decrease in serum BDNF is correlated with injury severity ${ }^{56}$ and poor recovery ${ }^{53}$. Recently, it has been shown that exogenous BDNF helps in mitigating neuronal metabolic defects and thereby improves neuronal survival after mechanical injury ${ }^{57}$. Protective effects of exogenous BDNF against neuronal apoptosis following mild ischemic brain injury has been shown by Galvin and Oorschot $(2003)^{58}$, whereas the commercially available drug, simvastatin, increased hippocampal BDNF level and neurogenesis in a rat model of $\mathrm{TBI}^{59}$. It is possible that neuropathological and behavioral changes after TBI observed in this study were mediated, at least partially, through BDNF. One of the major mechanisms by which hMSCs exert their effects in CNS repair and regeneration is by secreting neurotrophins $s^{52,60,61}$. Following transplantation, these cells may directly influence neuronal repair or stimulate the glial cells to secrete neurotrophins like BDNF or Nerve Growth Factor. On the other hand, the interaction between glial cells and hMSCs may lead to neurotrophin release and subsequent neuronal repair ${ }^{61,62}$. In this study, we observed that combination treatment, which showed the best recovery of histological, behavioral and sensorimotor parameters, also had significantly increased BDNF levels in serum as compared to the TBI or vehicle groups. Thus, it clearly indicates at least partial involvement of BDNF in the combination treatment induced recovery mechanism.

In stem cell therapy of brain injury, a key step in recovery is neuro-regeneration. In vitro differentiation of embryonic stem cells to neural cells has been shown by Guan et al. ${ }^{63}$. Mahmood et al. have shown that hMSC injected in the rat after TBI promotes tissue repair through neurogenesis and synaptogenesis ${ }^{64}$. Adults have two major neural stem cell niches, the SVZ and the dentate gyrus ${ }^{65}$. We observed that both hMSC and combination treatment enhanced neurogenesis in these regions. But the extent of neurogenesis reached the level of the sham animals only in the combination therapy group, which clearly showed the efficacy of this therapeutic strategy. Since BDNF has a potent role in neuroprotection and neurogenesis ${ }^{66}$, it is possible that hMSC induced neuroprotective and neurogenic effects were mediated, at least partially, through BDNF. BDNF along with other neurotrophic factors has been shown to utilize the canonical $\beta$-catenin pathway to promote hMSC neurogenesis and synaptogenesis ${ }^{67}$. The specific mechanism of PPAR $\gamma$ activation in the improvement of hMSC functioning and hMSC-induced neurogenesis needs further investigation.

In summary, as shown in Fig. 6, FPI induced injury in rat brains with significant tissue loss and neurodegeneration which persisted at $35 \mathrm{dpi}$. TBI in rats induced microglial and astroglial activation, increased secretion of proinflammatory cytokines and caused behavioral and sensorimotor deficits. PG reduced neuroinflammation in the brain by decreasing inflammatory cytokine production prior to hMSC transplantation. Reduction of local cerebral inflammation improved the efficacy of transplanted hMSC which was evident by increased neurogenesis, reduced anxiety-like behavior and reduced pain sensation in combination treated rats. Possibly, in a reduced inflammatory microenvironment, hMSCs helped in histological and behavioral recovery through enhanced production of neurotrophic factors like BDNF. Thus, in this study, we have shown that using PG before hMSC transplantation is a better therapeutic strategy for treating TBI compared to hMSC transplantation alone. 


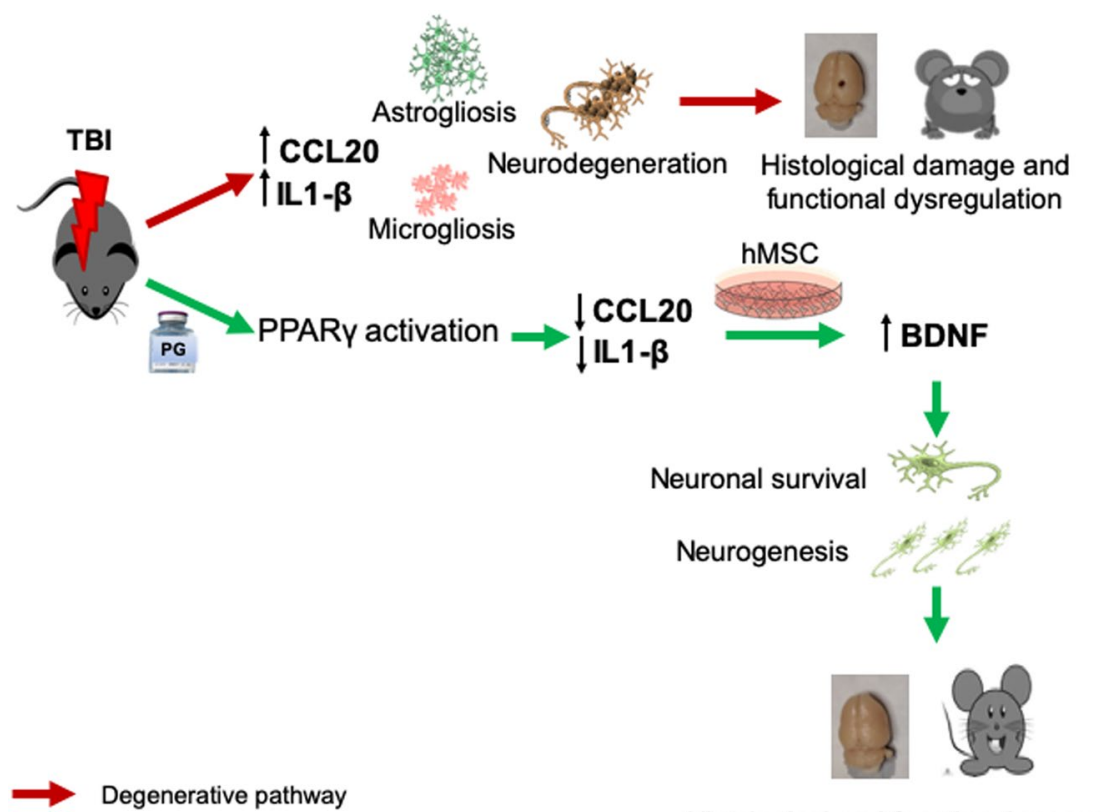

$\rightarrow$ Degenerative pathway

Histological and functional recovery

Figure 6. Schematic representation summarizing the effect of Pioglitazone (PG) and hMSC combination treatment on improving outcomes in rats after traumatic brain injury (TBI). TBI induces neurodegeneration and evokes inflammatory reactions including elevated cytokines CCL20 and IL1- $\beta$, microgliosis and astrogliosis. These lead to histological and functional deficits. PG treatment after TBI activates PPAR $\gamma$ and reduces CCL2 2 and IL1- $\beta$. In the reduced inflammatory microenvironment hMSCs increased BDNF secretion which at least partially improves the histological and functional recovery. CCL20, Chemokine ligand protein 20, IL1- $\beta$, Interleukin 1 beta, hMSC, human mesenchymal stem cells, BDNF, brain-derived neurotrophic factor.

\section{Methods}

Animals and TBI induction. All animal procedures were conducted in accordance with the NIH Guide for the Care and Use of Laboratory Animals and approved by the Institutional Animal Care and Use Committee of the University of South Florida. Forty male Sprague-Dawley rats (Envigo, USA) weighing 250 to $300 \mathrm{~g}$ were housed in a climate-controlled facility with food and water available ad libitum. Animals in this study were divided into 6 groups: 1 . Sham $(n=8), 2$. TBI $(n=6)$, 3. Vehicle treated (TBI + DMSO, $n=5), 4$. PG treated $(T B I+P G, n=8)$, 5. hMSC treated (TBI + hMSC, $\mathrm{n}=6), 6$. Combination treatment (Combo.) $(\mathrm{TBI}+\mathrm{PG}+\mathrm{hMSC}, \mathrm{n}=7)$.

Animals were anesthetized using a mixture of ketamine $(90 \mathrm{mg} / \mathrm{kg})$ and xylazine $(10 \mathrm{mg} / \mathrm{kg})$ (intraperitoneal, i.p.). Carprofen $(5 \mathrm{mg} / \mathrm{kg}$, subcutaneous, s.c., pre-operative and post-operative up to $48 \mathrm{~h}$ ) and buprenorphine ( $5 \mathrm{mg} / \mathrm{kg}$, s.c., pre-operative) were administered to all animals. Body temperature was monitored and maintained using a heating pad during the entire surgical procedure. Using a stereotaxic frame, a $1 \mathrm{~mm}$ diameter craniotomy was performed with a $0.7 \mathrm{~mm}$ drill bit, $2 \mathrm{~mm}$ lateral and $2.3 \mathrm{~mm}$ caudal to the bregma on the right side of the midline ${ }^{11}$. In case of accidental disruption of the meninges, the animal was excluded from the study. A female luer-lock hub was implanted at the craniotomy site, secured with dental cement and connected to fluid percussion injury (FPI) device. An impact ranging from 2.5-3.0 atm. was administered. Sham animals underwent all surgical procedures except the impact delivery. The luer-lock was detached, the craniotomy hole sealed with bone wax, and the scalp was sutured. Rats were returned to their home cages and allowed to recover for 35 days prior to subsequent experiments.

Drug and cell administration. Pioglitazone hydrochloride (PG) (Tocris, Inc.) dissolved in dimethyl sulfoxide (DMSO) at a dose of $2 \mathrm{mg} / \mathrm{kg}$ in $100 \mathrm{uL}$ or equal volume of vehicle was injected i.p. once a day for 5 days after TBI.

hMSCs were obtained from the Institute for Regenerative Medicine, Texas A\&M Health Science Center. Cells were cultured in $\alpha$ Minimum Essential Medium ( $\alpha$ MEM) (Gibco, cat\#12561-056) supplemented with 16.5\% FBS, $2 \mathrm{mM}$ L-glutamine and $1 \%$ penicillin and streptomycin at $37^{\circ} \mathrm{C}$ and $5 \% \mathrm{CO}_{2}$. At $90-95 \%$ confluence, cells were detached, washed, and resuspended in sterile phosphate buffered saline (PBS). The final volume of the cell suspension was adjusted so that $1 \times 10^{6}$ live cells were present in $100 \mu \mathrm{L}$ of cell suspension. On day 5 post impact, hyaluronidase was applied to each nostril (100U) and after $30 \mathrm{~min}$ hMSCs were instilled through the nostrils $(50 \mu \mathrm{L} /$ nostril) under isoflurane anesthesia. Rats were allowed to recover on a heating pad and returned to their home cage. hMSCs labeled with $1 \mu \mathrm{g} / \mathrm{mL}$ DiR (XenoLight DiR, Caliper Lifesciences) were washed and resuspended in the required volume of sterile PBS. Rats were injected with $1 \times 10^{6} \mathrm{hMSCs}$ either through the tail vein (i.v.) in $200 \mu \mathrm{l}$ or by the i.n. route $50 \mu \mathrm{l}$ per nostril. Animals were imaged after 7 days with the IVIS system using $710 \mathrm{~nm}$ excitation and $760 \mathrm{~nm}$ emission. Radiant efficiency (photons per second per square centimeter per steridian divided by microwatts per square centimeter $\left(\left(\mathrm{p} / \mathrm{s} / \mathrm{cm}^{2} / \mathrm{sr}\right) /\left(\mu \mathrm{W} / \mathrm{cm}^{2}\right)\right)$ of the region of interest (ROI) was plotted for each organ and each method of administration. 
Open field test (OFT). After 7 days of acclimation and gentle handling, baseline activity in the open field arena $(90 \mathrm{~cm}(\mathrm{~W}) \times 90 \mathrm{~cm}(\mathrm{D}) \times 40 \mathrm{~cm}(\mathrm{H}) \mathrm{cm}$ enclosure) was recorded for $10 \mathrm{~min}$ using Noldus Ethovision XT 10 software. The OFT was repeated for $10 \mathrm{~min}$ on $35 \mathrm{dpi}$. Rats were gently placed in the center of the arena and allowed $2 \mathrm{~min}$ to acclimatize in the arena before the start of the recording. The arena was cleaned with $70 \%$ ethyle alcohol and dried between rats. Activities in the peripheral and central zone were analyzed. Grooming and rearing behaviors were analyzed independently by 3 people blinded to the experimental conditions and averaged.

Cold plantar assay. The Cold plantar assay was performed to assess sensorimotor behavior. On 35 dpi rats were held gently in the lap by one experimenter with the hind paws hanging freely. A small portion of powdered dry ice compacted in the shape of a stick was pushed out from the cylinder of a syringe with the plunger and wrapped in a nitrile glove. The wrapped dry ice was touched to the plantar of the freely hanging hind paws, one paw at a time and the time of paw withdrawal was recorded. Care was taken that the paw movement was not hindered by any means. An additional group of naïve animals served as controls.

Euthanasia, tissue harvest, and processing. On $35 \mathrm{dpi}$ animals were deeply anesthetized with ketamine $(75 \mathrm{mg} / \mathrm{kg})$ and xylazine $(7.5 \mathrm{mg} / \mathrm{kg})$. Blood was collected and rats were perfused with $0.9 \%$ saline followed by $4 \%$ paraformaldehyde (PFA) in $0.1 \mathrm{M}$ phosphate buffer ( $\mathrm{pH} 7.4$ ). The brains were post-fixed in $2 \% \mathrm{PFA}$, saturated with sucrose solution, frozen, and $30 \mu \mathrm{m}$ coronal cryo-sections were generated.

Thionin and Fluorojade staining. Sections were treated with $1.25 \%$ thionin acetate solution (Sigma Aldrich, USA) for 45 seconds followed by rinsing with deionized water for $1 \mathrm{~min}$., dehydrated through graded ethanol, cleared with xylene, and coverslipped with DPX mounting medium (Electron Microscopy Sciences, Ft. Washington, PA). For fluorojade (FJ) staining ${ }^{68,69}$ slide-mounted sections were hydrated in graded ethanol and then oxidized with $0.06 \% \mathrm{KMnO} 4$ solution for 15 minutes and stained in a $0.001 \%$ solution of FJ (Histochem, Jefferson, AR) in $0.1 \%$ acetic acid for $30 \mathrm{~min}$. Slides were rinsed, dried at $45^{\circ} \mathrm{C}$ for $20 \mathrm{~min}$, cleared with xylene, and coverslipped using DPX mounting medium.

Immunohistochemistry. For immune-peroxidase staining, sections were incubated in 3\% hydrogen peroxide for $20 \mathrm{~min}$, washed with PBS and heated in antigen unmasking solution (1:100; Vector Laboratories Inc., Burlingame, CA) for $40 \mathrm{~min}$ at $90^{\circ} \mathrm{C}$, cooled, permeabilized for $1 \mathrm{~h}$ in permeabilization buffer $(10 \%$ host serum, $0.1 \%$ Triton X-100 in PBS) and incubated overnight at $4^{\circ} \mathrm{C}$ with primary antibody in antibody solution (5\% host serum, $0.05 \%$ Triton X-100 in PBS). Next day, sections were washed with PBS, incubated sequentially with biotinylated secondary antibody $(2 \mathrm{~h}$, room temperature $(\mathrm{RT}))$ and avidin-biotin complex mixture (ABC,1:100; Vector Laboratories, Inc., Burlingame, Ca) (1 h, RT) and developed using DAB/peroxide solution (Vector Laboratories, Inc.). After 3 washes, sections were dried, dehydrated, cleared with xylene and coverslipped with DPX. For fluorescence immunohistochemistry, no peroxide blocking was performed. After primary antibody incubation, sections were incubated with fluorescent secondary antibody, washed with PBS, dried and cover slipped with vectashield anti-fade mounting medium with DAPI. Detailed information on antibodies used in this study is shown in Table S1. Sections were viewed with an Olympus IX71 microscope using appropriate filters. Images were captured using an Olympus DP70 imaging system. The low magnification (4x) collages of the entire brain sections were taken and processed with a Keyence BZ-X800 microscope and associated software (Keyence America).

Enzyme Linked Immunosorbent Assay (ELISA). Serum samples were thawed on ice for ELISA development using Picokine rat BDNF ELISA kit from myBiosource (San Diego, USA, Cat\# MBS175935) following manufacturer's instruction. Briefly, standards or samples were added to wells of a 96 well ELISA plate pre-coated with anti-BDNF antibody and incubated overnight at $4{ }^{\circ} \mathrm{C}$. The plate was washed, and incubated sequentially with biotinylated anti-rat BDNF antibody for $1 \mathrm{~h}$, avidin-biotin-peroxidase complex for $30 \mathrm{~min}$ and color development reagent for $25 \mathrm{~min}$ at room temperature. Reactions were stopped with $2 \mathrm{~N} \mathrm{H}_{2} \mathrm{SO}_{4}$. The absorbance readings were taken at $450 \mathrm{~nm}$ using a Synergy H4 hybrid reader (BioTek). Serum BDNF concentration was expressed as pg/mL.

Image analysis and quantitation. Cell count or intensity was calculated using the NIH Image J software. Images $(100 \mathrm{x}$ or $200 \mathrm{x})$ were taken at the same exposure and digital gain settings for a given magnification for all the sections in order to eliminate differential background intensity and/or false positive signal. The RGB channels of fluorescent images were split and either the red or green channel was used for Quantitation. The bright field images were converted to grey scale. The brightness and contrast were adjusted to discard the noise pixels. The threshold of the binary images was adjusted between 0 and 255 to highlight all positive cells to be counted. In the set measurement tool, the particle sizes were adjusted to exclude the small noise pixels or the large clumps from the count. Circularity was adjusted between 0 and 1 to discard any cell fragments, cell processes, or tissue aggregates that can create false results. The same specifications were used to quantitate across the board. The number of cells was counted using Analyze $>$ Particle tool. The fluorescence intensity (integrated density) was measured using Analyze $>$ Measure tool. For lesion volume measurement, the scale was calibrated using the set scale tool, the horizontal and vertical diameters of the lesion area were measured and averaged. The lesion area from each section was calculated using the average diameter and multiplied by the section thickness $(30 \mu)$ to calculate the lesion volume for each section. The values were then added up to obtain total lesion volume from each brain and as expressed as $\mathrm{mm}^{3}$.

Statistical analysis. All data are presented as mean \pm Standard Error of Mean (S.E.M.). Statistical significance was evaluated by one-way ANOVA with a Tukey's or Fisher's post-hoc test or student's t-test. A p value of less than 0.05 was considered statistically significant for all comparisons. 


\section{References}

1. Hyder, A. A., Wunderlich, C. A., Puvanachandra, P., Gururaj, G. \& Kobusingye, O. C. The impact of traumatic brain injuries: a global perspective. NeuroRehabilitation 22, 341-353 (2007).

2. Langlois, J. A., Rutland-Brown, W. \& Wald, M. M. The epidemiology and impact of traumatic brain injury: a brief overview. J Head Trauma Rehabil 21, 375-378 (2006).

3. CDC, N. C. f. I. P. a. C. Rates of TBI-related Emergency Department Visits, Hospitalizations, and Deaths - United States, 2001-2010 (2016).

4. Nichol, A. et al. Erythropoietin in traumatic brain injury (EPO-TBI): a double-blind randomised controlled trial. Lancet 386, 2499-2506, https://doi.org/10.1016/S0140-6736(15)00386-4 (2015).

5. Robertson, C. S. et al. Effect of erythropoietin and transfusion threshold on neurological recovery after traumatic brain injury: a randomized clinical trial. JAMA 312, 36-47, https://doi.org/10.1001/jama.2014.6490 (2014).

6. Skolnick, B. E. et al. A clinical trial of progesterone for severe traumatic brain injury. N Engl J Med 371, 2467-2476, https://doi. org/10.1056/NEJMoa1411090 (2014).

7. Galindo, L. T. et al. Mesenchymal stem cell therapy modulates the inflammatory response in experimental traumatic brain injury. Neurol Res Int 2011, 564089, https://doi.org/10.1155/2011/564089 (2011).

8. Parr, A. M., Tator, C. H. \& Keating, A. Bone marrow-derived mesenchymal stromal cells for the repair of central nervous system injury. Bone Marrow Transplant 40, 609-619, https://doi.org/10.1038/sj.bmt.1705757 (2007).

9. Redondo-Castro, E. et al. Interleukin-1 primes human mesenchymal stem cells towards an anti-inflammatory and pro-trophic phenotype in vitro. Stem Cell Res Ther 8, 79, https://doi.org/10.1186/s13287-017-0531-4 (2017).

10. Garcia-Olmo, D. et al. A phase I clinical trial of the treatment of Crohn's fistula by adipose mesenchymal stem cell transplantation. Dis Colon Rectum 48, 1416-1423, https://doi.org/10.1007/s10350-005-0052-6 (2005).

11. Das, M. et al. Lateral fluid percussion injury of the brain induces CCL20 inflammatory chemokine expression in rats. $J$ Neuroinflammation 8, 148, https://doi.org/10.1186/1742-2094-8-148 (2011).

12. Leonardo, C. C. et al. CCL20 Is Associated with Neurodegeneration Following Experimental Traumatic Brain Injury and Promotes Cellular Toxicity In Vitro. Transl Stroke Res 3, 357-363, https://doi.org/10.1007/s12975-012-0203-8 (2012).

13. Khodeer, D. M., Zaitone, S. A., Farag, N. E. \& Moustafa, Y. M. Cardioprotective effect of pioglitazone in diabetic and non-diabetic rats subjected to acute myocardial infarction involves suppression of AGE-RAGE axis and inhibition of apoptosis. Can J Physiol Pharmacol 94, 463-476, https://doi.org/10.1139/cjpp-2015-0135 (2016).

14. Qi, W. et al. The roles of Kruppel-like factor 6 and peroxisome proliferator-activated receptor-gamma in the regulation of macrophage inflammatory protein-3alpha at early onset of diabetes. Int J Biochem Cell Biol 43, 383-392, https://doi.org/10.1016/j. biocel.2010.11.008 (2011).

15. Glatz, T. et al. Peroxisome-proliferator-activated receptors gamma and peroxisome-proliferator-activated receptors beta/delta and the regulation of interleukin 1 receptor antagonist expression by pioglitazone in ischaemic brain. J Hypertens 28, 1488-1497, https:// doi.org/10.1097/HJH.0b013e3283396e4e (2010).

16. Kobayashi, T. et al. Pioglitazone, a peroxisome proliferator-activated receptor gamma agonist, reduces the progression of experimental osteoarthritis in guinea pigs. Arthritis Rheum 52, 479-487, https://doi.org/10.1002/art.20792 (2005).

17. Zhang, W. Y., Schwartz, E. A., Permana, P. A. \& Reaven, P. D. Pioglitazone inhibits the expression of inflammatory cytokines from both monocytes and lymphocytes in patients with impaired glucose tolerance. Arterioscler Thromb Vasc Biol 28, 2312-2318, https:// doi.org/10.1161/ATVBAHA.108.175687 (2008).

18. da Silva Meirelles, L., Chagastelles, P. C. \& Nardi, N. B. Mesenchymal stem cells reside in virtually all post-natal organs and tissues. J Cell Sci 119, 2204-2213, https://doi.org/10.1242/jcs.02932 (2006).

19. Karp, J. M. \& Leng Teo, G. S. Mesenchymal stem cell homing: the devil is in the details. Cell Stem Cell 4, 206-216, https://doi. org/10.1016/j.stem.2009.02.001 (2009).

20. Hasan, A. et al. Mesenchymal Stem Cells in the Treatment of Traumatic Brain Injury. Front Neurol 8, 28, https://doi.org/10.3389/ fneur.2017.00028 (2017).

21. Pittenger, M. F. et al. Multilineage potential of adult human mesenchymal stem cells. Science 284, 143-147 (1999).

22. Sanchez-Ramos, J. et al. Adult bone marrow stromal cells differentiate into neural cells in vitro. Exp Neurol 164, 247-256, https://doi. org/10.1006/exnr.2000.7389(2000).

23. Alexanian, A. R., Fehlings, M. G., Zhang, Z. \& Maiman, D. J. Transplanted neurally modified bone marrow-derived mesenchymal stem cells promote tissue protection and locomotor recovery in spinal cord injured rats. Neurorehabil Neural Repair 25, 873-880, https://doi.org/10.1177/1545968311416823(2011).

24. Chamberlain, G., Fox, J., Ashton, B. \& Middleton, J. Concise review: mesenchymal stem cells: their phenotype, differentiation capacity, immunological features, and potential for homing. Stem Cells 25, 2739-2749, https://doi.org/10.1634/stemcells.2007-0197 (2007).

25. Chamberlain, G., Smith, H., Rainger, G. E. \& Middleton, J. Mesenchymal stem cells exhibit firm adhesion, crawling, spreading and transmigration across aortic endothelial cells: effects of chemokines and shear. PLoS One 6, e25663, https://doi.org/10.1371/journal. pone.0025663 (2011).

26. Wu, J. et al. Intravenously administered bone marrow cells migrate to damaged brain tissue and improve neural function in ischemic rats. Cell Transplant 16, 993-1005 (2008).

27. Xiong, Y., Mahmood, A. \& Chopp, M. Emerging potential of exosomes for treatment of traumatic brain injury. Neural Regen Res 12, 19-22, https://doi.org/10.4103/1673-5374.198966 (2017).

28. Cui, G. H. et al. Exosomes derived from hypoxia-preconditioned mesenchymal stromal cells ameliorate cognitive decline by rescuing synaptic dysfunction and regulating inflammatory responses in APP/PS1 mice. FASEB J 32, 654-668, https://doi. org/10.1096/fj.201700600R (2018).

29. Wei, L., Wei, Z. Z., Jiang, M. Q., Mohamad, O. \& Yu, S. P. Stem cell transplantation therapy for multifaceted therapeutic benefits after stroke. Prog Neurobiol. https://doi.org/10.1016/j.pneurobio.2017.03.003 (2017).

30. Danielyan, L. et al. Intranasal delivery of cells to the brain. Eur J Cell Biol 88, 315-324, https://doi.org/10.1016/j.ejcb.2009.02.001 (2009).

31. Badiavas, E. V. \& Falanga, V. Treatment of chronic wounds with bone marrow-derived cells. Arch Dermatol 139, 510-516, https:// doi.org/10.1001/archderm.139.4.510 (2003).

32. Suzuki, K. et al. Role of interleukin-1beta in acute inflammation and graft death after cell transplantation to the heart. Circulation 110, II219-224, https://doi.org/10.1161/01.CIR.0000138388.55416.06 (2004).

33. Molcanyi, M. et al. Trauma-associated inflammatory response impairs embryonic stem cell survival and integration after implantation into injured rat brain. J Neurotrauma 24, 625-637, https://doi.org/10.1089/neu.2006.0180 (2007).

34. Geesala, R., Dhoke, N. R. \& Das, A. Cox-2 inhibition potentiates mouse bone marrow stem cell engraftment and differentiationmediated wound repair. Cytotherapy 19, 756-770, https://doi.org/10.1016/j.jcyt.2017.03.072 (2017).

35. Arai, K., Jin, G., Navaratna, D. \& Lo, E. H. Brain angiogenesis in developmental and pathological processes: neurovascular injury and angiogenic recovery after stroke. FEBS J 276, 4644-4652, https://doi.org/10.1111/j.1742-4658.2009.07176.x (2009).

36. Culman, J., Zhao, Y., Gohlke, P. \& Herdegen, T. PPAR-gamma: therapeutic target for ischemic stroke. Trends Pharmacol Sci 28, 244-249, https://doi.org/10.1016/j.tips.2007.03.004 (2007). 
37. Villapol, S. Roles of Peroxisome Proliferator-Activated Receptor Gamma on Brain and Peripheral Inflammation. Cell Mol Neurobiol 38, 121-132, https://doi.org/10.1007/s10571-017-0554-5 (2018).

38. Heneka, M. T. \& Landreth, G. E. PPARs in the brain. Biochim Biophys Acta 1771, 1031-1045, https://doi.org/10.1016/j. bbalip.2007.04.016 (2007).

39. Vallee, A. \& Lecarpentier, Y. Crosstalk Between Peroxisome Proliferator-Activated Receptor Gamma and the Canonical WNT/betaCatenin Pathway in Chronic Inflammation and Oxidative Stress During Carcinogenesis. Front Immunol 9, 745, https://doi. org/10.3389/fimmu.2018.00745 (2018).

40. Jones, N. C. et al. Experimental traumatic brain injury induces a pervasive hyperanxious phenotype in rats. J Neurotrauma 25, 1367-1374, https://doi.org/10.1089/neu.2008.0641 (2008).

41. Kim, H. et al. Treatment of traumatic brain injury with 17alpha-ethinylestradiol-3-sulfate in a rat model. J Neurosurg 127, 23-31, https://doi.org/10.3171/2016.7.JNS161263 (2017).

42. Rowe, R. K. et al. Diffuse traumatic brain injury induces prolonged immune dysregulation and potentiates hyperalgesia following a peripheral immune challenge. Mol Pain 12, https://doi.org/10.1177/1744806916647055 (2016).

43. Lahz, S. \& Bryant, R. A. Incidence of chronic pain following traumatic brain injury. Arch Phys Med Rehabil 77, 889-891 (1996).

44. Gironda, R. J., Clark, M. E., Massengale, J. P. \& Walker, R. L. Pain among veterans of Operations Enduring Freedom and Iraqi Freedom. Pain Med 7, 339-343, https://doi.org/10.1111/j.1526-4637.2006.00146.x (2006).

45. Feliciano, D. P. et al. Nociceptive sensitization and BDNF up-regulation in a rat model of traumatic brain injury. Neurosci Lett 583, 55-59, https://doi.org/10.1016/j.neulet.2014.09.030 (2014).

46. Elliott, M. B., Oshinsky, M. L., Amenta, P. S., Awe, O. O. \& Jallo, J. I. Nociceptive neuropeptide increases and periorbital allodynia in a model of traumatic brain injury. Headache 52, 966-984, https://doi.org/10.1111/j.1526-4610.2012.02160.x (2012).

47. Old, E. A., Clark, A. K. \& Malcangio, M. The role of glia in the spinal cord in neuropathic and inflammatory pain. Handb Exp Pharmacol 227, 145-170, https://doi.org/10.1007/978-3-662-46450-2_8 (2015).

48. Wagner, B. et al. Neuronal survival depends on EGFR signaling in cortical but not midbrain astrocytes. EMBO J 25, 752-762, https:// doi.org/10.1038/sj.emboj.7600988 (2006).

49. Sofroniew, M. V. Molecular dissection of reactive astrogliosis and glial scar formation. Trends Neurosci 32, 638-647, https://doi. org/10.1016/j.tins.2009.08.002 (2009).

50. Phatnani, H. \& Maniatis, T. Astrocytes in neurodegenerative disease. Cold Spring Harb Perspect Biol 7, https://doi.org/10.1101/ cshperspect.a020628 (2015).

51. Rojas, M. et al. Bone marrow-derived mesenchymal stem cells in repair of the injured lung. Am J Respir Cell Mol Biol 33, 145-152, https://doi.org/10.1165/rcmb.2004-0330OC (2005).

52. Kim, H. J., Lee, J. H. \& Kim, S. H. Therapeutic effects of human mesenchymal stem cells on traumatic brain injury in rats: secretion of neurotrophic factors and inhibition of apoptosis. J Neurotrauma 27, 131-138, https://doi.org/10.1089/neu.2008-081810.1089/ neu.2008.0818 (2010)

53. Korley, F. K. et al. Circulating Brain-Derived Neurotrophic Factor Has Diagnostic and Prognostic Value in Traumatic Brain Injury. J Neurotrauma 33, 215-225, https://doi.org/10.1089/neu.2015.3949 (2016).

54. Martinowich, K., Manji, H. \& Lu, B. New insights into BDNF function in depression and anxiety. Nat Neurosci 10, 1089-1093, https://doi.org/10.1038/nn1971 (2007).

55. Griesbach, G. S., Hovda, D. A. \& Gomez-Pinilla, F. Exercise-induced improvement in cognitive performance after traumatic brain injury in rats is dependent on BDNF activation. Brain Res 1288, 105-115, https://doi.org/10.1016/j.brainres.2009.06.045 (2009).

56. Kalish, H. \& Phillips, T. M. Analysis of neurotrophins in human serum by immunoaffinity capillary electrophoresis (ICE) following traumatic head injury. J Chromatogr B Analyt Technol Biomed Life Sci 878, 194-200, https://doi.org/10.1016/j.jchromb.2009.10.022 (2010).

57. Xu, Z., Lv, X. A., Dai, Q., Lu, M. \& Jin, Z. Exogenous BDNF Increases Mitochondrial pCREB and Alleviates Neuronal Metabolic Defects Following Mechanical Injury in a MPTP-Dependent Way. Mol Neurobiol 55, 3499-3512, https://doi.org/10.1007/s12035017-0576-5 (2018).

58. Galvin, K. A. \& Oorschot, D. E. Continuous low-dose treatment with brain-derived neurotrophic factor or neurotrophin-3 protects striatal medium spiny neurons from mild neonatal hypoxia/ischemia: a stereological study. Neuroscience 118, 1023-1032 (2003).

59. Wu, H. et al. Simvastatin-mediated upregulation of VEGF and BDNF, activation of the PI3K/Akt pathway, and increase of neurogenesis are associated with therapeutic improvement after traumatic brain injury. J Neurotrauma 25, 130-139, https://doi. org/10.1089/neu.2007.0369 (2008).

60. Chen, X. et al. Ischemic rat brain extracts induce human marrow stromal cell growth factor production. Neuropathology 22, 275-279 (2002).

61. Li, Y. et al. Human marrow stromal cell therapy for stroke in rat: neurotrophins and functional recovery. Neurology 59, 514-523 (2002).

62. Hefti, F. Nerve growth factor promotes survival of septal cholinergic neurons after fimbrial transections. J Neurosci 6, 2155-2162 (1986).

63. Guan, K., Chang, H., Rolletschek, A. \& Wobus, A. M. Embryonic stem cell-derived neurogenesis. Retinoic acid induction and lineage selection of neuronal cells. Cell Tissue Res 305, 171-176 (2001).

64. Mahmood, A., Lu, D., Qu, C., Goussev, A. \& Chopp, M. Human marrow stromal cell treatment provides long-lasting benefit after traumatic brain injury in rats. Neurosurgery $57,1026-1031$; discussion 1026-1031 (2005).

65. Doeppner, T. R. et al. Post-stroke transplantation of adult subventricular zone derived neural progenitor cells-A comprehensive analysis of cell delivery routes and their underlying mechanisms. Exp Neurol 273, 45-56, https://doi.org/10.1016/j. expneurol.2015.07.023 (2015).

66. Vithlani, M. et al. The ability of BDNF to modify neurogenesis and depressive-like behaviors is dependent upon phosphorylation of tyrosine residues 365/367 in the GABA(A)-receptor gamma2 subunit. J Neurosci 33, 15567-15577, https://doi.org/10.1523/ JNEUROSCI.1845-13.2013 (2013)

67. Tsai, H. L. et al. Wnts enhance neurotrophin-induced neuronal differentiation in adult bone-marrow-derived mesenchymal stem cells via canonical and noncanonical signaling pathways. PLoS One 9, e104937, https://doi.org/10.1371/journal.pone.0104937 (2014).

68. Schmued, L. C., Albertson, C. \& Slikker, W. Jr. Fluoro-Jade: a novel fluorochrome for the sensitive and reliable histochemical localization of neuronal degeneration. Brain Res 751, 37-46 (1997).

69. Duckworth, E. A. et al. Temporary focal ischemia in the mouse: technical aspects and patterns of Fluoro-Jade evident neurodegeneration. Brain Res 1042, 29-36, https://doi.org/10.1016/j.brainres.2005.02.021 (2005).

\section{Acknowledgements}

This work is supported by a Veterans Affairs Merit Review grant (BX002668) to Subhra Mohapatra, and Research Career Scientist Awards to Dr. Subhra Mohapatra (IK6BX004212) and Dr. Shyam Mohapatra (IK6 BX003778). Though this report is based upon work supported, in part, by the Department of Veterans Affairs, Veterans Health Administration, Office of Research and Development, the contents of this report do not represent the views of the Department of Veterans Affairs or the United States Government. We acknowledge the extensive help of Dr. Edwin Weeber and Ms. Melinda Peters in behavioral testing of rats in this study. 


\section{Author Contributions}

S.M., S.S.M. and M.D. designed the experiments. M.D., K.M., X.T., J.H. and E.F. performed experiments. M.D. analyzed data and wrote the manuscript. S.M., S.S.M. and A.W. reviewed and edited the manuscript. All authors have seen and approved the manuscript.

\section{Additional Information}

Supplementary information accompanies this paper at https://doi.org/10.1038/s41598-019-49428-y.

Competing Interests: The authors declare no competing interests.

Publisher's note Springer Nature remains neutral with regard to jurisdictional claims in published maps and institutional affiliations.

(c) (i) Open Access This article is licensed under a Creative Commons Attribution 4.0 International License, which permits use, sharing, adaptation, distribution and reproduction in any medium or format, as long as you give appropriate credit to the original author(s) and the source, provide a link to the Creative Commons license, and indicate if changes were made. The images or other third party material in this article are included in the article's Creative Commons license, unless indicated otherwise in a credit line to the material. If material is not included in the article's Creative Commons license and your intended use is not permitted by statutory regulation or exceeds the permitted use, you will need to obtain permission directly from the copyright holder. To view a copy of this license, visit http://creativecommons.org/licenses/by/4.0/.

(C) The Author(s) 2019 